\title{
Burkitt's lymphoma patients in Northwest Cameroon have a lower incidence of sickle cell trait (Hb AS) than healthy controls
}

\author{
P B Hesseling, ${ }^{1}$ MD; D T Jam, ${ }^{2}$ MD; D D Palmer, ${ }^{2}$ MD; P Wharin,,${ }^{3}$ MD; G S Tuh, ${ }^{2}$ Dip Tech; R Bardin,,${ }^{2}$ MD; M Kidd, ${ }^{4} \mathrm{PhD}$ \\ ${ }^{1}$ Department of Paediatrics and Child Health, Tygerberg Children's Hospital, Stellenbosch University, Cape Town, South Africa \\ ${ }^{2}$ Mbingo Baptist Hospital, Mbingo, Northwest region, Cameroon \\ ${ }^{3}$ Beryl Thyer Memorial Africa Trust, Warkton, UK \\ ${ }^{4}$ Department of Biostatistics, Stellenbosch University, Cape Town, South Africa
}

Corresponding author: P B Hesseling (pbh@sun.ac.za)

Contradictory findings have been reported from Africa with regard to the risk of developing Burkitt's lymphoma (BL) in sickle cell trait (AS) carriers. Haemoglobin electrophoresis was performed in $78 \mathrm{BL}$ patients in the Northwest region of Cameroon, and in 78 nearest-neighbour controls of the same age, sex and tribe from the same village. AS was confirmed in 4 of 78 (5.13\%) BL patients and in 11 of 78 (14.10\%) controls $\left(\chi^{2}, p=0.052\right.$; Fisher's exact, one-tailed, $\left.p=0.050\right)$. Sickle cell trait carriers had a marginal statistically reduced risk of developing BL.

S Afr Med J 2016;106(7):686. DOI:10.7196/SAMJ.2016.v106i7.10693

Burkitt's lymphoma (BL), the most common childhood cancer in sub-Saharan Africa, occurs mainly in areas with holo-endemic malaria. ${ }^{[1]}$ Young children with haemoglobin (Hb) AS (sickle cell trait) are largely protected against severe Plasmodium falciparum malaria. ${ }^{[2]}$ The prevalence of AS in the Northwest region of Cameroon, a holo-endemic malaria area, is between $10 \%$ and $30 \% \cdot{ }^{[2]}$ The annual incidence of BL between 2003 and 2010 was $2.58 / 100000$ children $<15$ years of age, and the monthly average of malaria cases was 10938 in the dry season and 11499 in the rainy season. ${ }^{[3]}$ It is hypothesised that the AS trait lowers the risk of developing BL.

One hundred Nigerian Yoruba children with BL had a significantly lower incidence of the AS genotype than hospital controls. No difference in frequency of AS was, however, subsequently observed between 306 Kenyan children with BL and age-, ethnically and geographically matched controls. ${ }^{[4,5]}$

We observed an AS incidence of $7 \%$ in 167 BL patients treated in Cameroon, ${ }^{[6]}$ and subsequently conducted a prospective study in surviving $\mathrm{BL}$ patients resident in the Northwest region.

\section{Methods}

Seventy-nine BL patients in the Northwest region of Cameroon were visited at home, where a nearest-neighbour control of the same tribe, age and sex was identified. One parent of a patient with BL refused consent. An ethylenediaminetetra-acetic acid (EDTA) venous blood sample was obtained from the index patients and controls, and paper electrophoresis was performed at $\mathrm{pH} 8.9$ in boric and trisaminomethane (TRIS) buffer. Institutional review board approval and informed parental consent were obtained.

\section{Results}

The 79 BL patients included 41 girls and 38 boys aged 4 - 17 (mean 9.9) years. Controls had a similar age and gender distribution. Four (5.13\%) BL patients had AS compared with $11(14.10 \%)$ controls $\left(\chi^{2}(\mathrm{df}=1)=3.74, p=0.052\right.$; Fisher's exact, one-tailed, $p=0.050$ ) (Table 1).

Table 1. Observed frequencies of
Hb AA and Hb AS
\begin{tabular}{lllll}
\hline Subjects & Hb AA & Hb AS & Total \\
\hline Patients, $n$ & 74 & 4 & 78 \\
Patients, \% & 94.87 & 5.13 & \\
Controls, $n$ & 67 & 11 & 78 \\
Controls, \% & 85.9 & 14.10 & \\
Total & 141 & 15 & 156 \\
$\chi^{2}$ (df=1)=3.74, $p=0.052$; Fisher's exact, one-tailed, \\
$p=0.050$.
\end{tabular}

\section{Discussion}

The validity of the Nigerian study ${ }^{[4]}$ was later questioned because controls were not from the same tribe, region and village. It is not clear whether controls in the Kenyan study ${ }^{[5]}$ were carefully matched for age and if they were indeed nearest-neighbour controls. This is of critical importance because of large differences in the distribution of the AS gene.

\section{Conclusion}

The relatively small number of patients studied limits the statistical significance of our findings. This study of confirmed BL patients and very well-matched controls did, however, demonstrate that AS carriers in Cameroon probably have a reduced risk of developing BL. Further studies in this regard are justified.

Acknowledgement. We acknowledge laboratory support from the Cameroon Baptist Convention Health Board.

\footnotetext{
1. Rainey JJ, Mwando WO, Wairiumu P, Morman AMM, Wilson ML, Rochford R. Spatial distribution of Burkitt's lymphoma in Kenya and association with malaria risk. Trop Med Int Health 2007;12:936-943.

2. Tchana Sinou M. Antenatal screening of sickle cell disease. 8th Postgraduate Course for Training in Reproductive Medicine and Reproductive Biology. Geneva: Geneva Foundation for Medicd Reproductive Biology. Geneva: Geneva Foundation for Medical Education and Research, 2008. http:www.gfmer.ch/ENDO/ PGC_ network/antenatal_ screening_sickle_cell_Tchana.htm
(accessed 17 May 2016).

. Lewis N, Young J, Hesseling PB, McCormick P, Wright N. Epidemiology of Burkitt's lymphoma in Northwest Province Cameroon 2003 - 2010. Paediatr Int Child Health 2012;32:82 85. DOI:10.1179/2046905511Y.0000000016

. Williams AO. Haemoglobin genotypes, ABO blood groups, an Burkitt's tumour. J Med Genet 1966;3:177-179.

5. Mulama DH, Bailey JA, Foley J, et al. Sickle cell trait is not associated with endemic Burkitt lymphoma: An ethnicity and malaria endemicity-matched case-control study suggests factors controlling EBV may serve as a predictive biomarker for this pediatric cancer Int J Cancer 2014;134(3):645-653. DOI:10.1002/ijc.28378

6. Hesseling $\mathrm{PB}$. Wharin. Poster presentation at the Africa International Society of Paediatric Oncology (SIOP) Conference, Cape Town, South Africa, 21 - 23 March 2012.
} 